\title{
Mejorar el clima en la enseñanza de la lectura
}

\author{
Peter Winograd \\ Lynne A. Smith
}
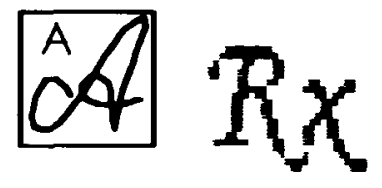

Tal vez temas como la motivación del alumno o la mejora del ambiente de trabajo sean relativamente sutiles, pero son extremadamente importantes. Si a la bora de plantear una reforma no les prestamos atención, si no trabajamos para mejorar el contexto en el que tiene lugar la enseñanza de la lectura, gran parte de nuestros conocimientos, técnicas, pruebas y tests acabarán por reforzar los viejos esquemas de comportamiento y, a largo plazo, todo seguirá igual.

Hay motivos para ser optimistas sobre la posibilidad de mejorar la alfabetización en este país... la última década ha sido testigo de avances sin precedentes en el conocimiento de los procesos básicos que tienen lugar en la lectura, su enseñanza y aprendizaje. Este conocimiento está hoy a nuestra disposición para mejorar los hábitos lectores en los EE.UU. (Anderson y otros, Becoming a nation of Readers, 1985, pág. 3).

Efectivamente, la información sobre la lectura ha aumentado sensiblemente tras las recientes investigaciones llevadas a cabo en este campo. El boom actual de artículos, ponencias, libros especializados sobre la lectura, en especial sobre la comprensión lectora, reflejan una auténtica evolución en la concepción de la lectura. El objeto de este artículo es examinar algunas de las actitudes que habrá que modificar si queremos ser testigos de una evolución paralela en el modo de enseñar a leer. Hemos seleccionado este tema porque creemos que la mejora de la enseñanza de la lectura exige algo más que una sólida comprensión del proceso lector, un repertorio de técnicas de enseñanza eficaces o estanterías repletas de material pedagógico

"Improving the climate for reading comprehension instruction" The Reading Teacher, diciembre 1987, 304-310. Reproducido con autorización de Peter Winograd y la International Reading Association.

(C) Traducción al castellano, CLEE, 1989 (Traducción de Begoña Jiménez. La I.R.A. no se responsabiliza de la fidelidad de la traducción). 
de calidad. Todo esto es básico, pero la mejora de la enseñanza de la lectura también requiere que cambie el clima en el que tiene lugar y que ejerce una fuerte influencia sobre lo que se enseña y se aprende en la mayoría de las aulas.

Tres aspectos interrelacionados del clima actual son especialmente importantes:

1. Un control o evaluación cuantitativista y la concepción reduccionista de la lectura que ello implica.

2. Los errores de carácter pasivo, unidos a una concepción de la lectura como actividad competitiva.

3. La enseñanza de la lectura y la pérdida de técnicas por parte del profesorado.

\section{CUANTITATIVISMO Y CONCEPCION REDUCCIONISTA DE LA LECTURA}

A primera vista, parece ser éste un procedimiento muy razonable para mejorar la educación en general y la enseñanza de la lectura en particular. Se definen los objetivos específicos y se procede a su seguimiento para comprobar si se han cumplido.

El problema surge a la hora de definir los objetivos y decidir cómo medirlos (Harper y Kilarr, 1978). La lectura es obviamente un proceso complejo, y definir sus objetivos no es tarea fácil. La variedad de posibles objetivos es abrumadora: desde conocer el nombre de las letras hasta hacer deducciones, desarrollar la metacognición o fomentar el gusto por la lectura.

En general los educadores, autoridades y ciudadanos americanos han atajado el problema reduciendo los objetivos de la lectura a metas sencillas y de medición poco costosa, con pruebas estándares a gran escala. No obstante, esta solución conduce a una concepción reduccionista de la lectura (Winograd y Greenlee, 1986), porque reduce los objetivos de la instrucción lectora, abandonando el objetivo de construir en los alumnos el amor por la lectura a cambio del simple desarrollo en los alumnos de un «nivel de competencia lectora identificable" (Shannon, 1983). Esta concepción reduccionista eștá presente en gran parte del material pedagógico. Las programaciones que acompañan al material de lectura, la lista de objetivos que incluyen casi todas las pruebas estandarizadas empleadas para evaluar el progreso de los niños y gran parte de las listas de técnicas esenciales vigentes en muchos estados reflejan esta concepción.

El motivo de que esté tan extendida deriva principalmente de que hace factible la cuantificación y verificación del cumplimiento de objetivos. Leer es un proceso complejo, pero si se reduce el objetivo a alcanzar una determinada puntuación en diversas pruebas, su cumplimiento no presenta dificultad. De este modo, el público cuenta con un criterio simple para evaluar el trabajo escolar de profesores y alumnos.

No pretendemos atacar la preocupación ciudadana sobre la calidad de la educación, sino la falsa sensación de seguridad que proporcionan estos métodos evaluadores. Es una seguridad falsa por diversas razones, dos de las cuales son especialmente importantes. La primera de ellas es que los métodos que imponen el cumplimiento de objetivos se basan en una concep- 
ción de la lectura incorrecta: la lectura como serie jerárquica de técnicas menores. Sin embargo, en agudo contraste con esta concepción se sitúan las tendencias actuales, que se centran èn «cómo el lector elabora el significado a partir de la forma impresa; qué experiencias, conocimientos, técnicas y motivación aporta el lector a la situación; cómo se presenta la información en el texto escrito, y la influencia que ejerce el contexto en el rendimiento" (Wixson y Peters, próxima publicación).

Es importante resaltar que las técnicas definidas en la mayor parte de los métodos cuantitativos tradicionales de cumplimiento de objetivos no constituyen técnicas menores en el sentido teórico de componentes independientes, verificables en el proceso lector (Rosenshine, 1980), sino que son intentos pragmáticos de identificar aspectos de la lectura en los que poder centrar la enseñanza y la evaluación.

De la investigación actual se desprende que tiene sentido emplear algunas de estas técnicas como parte de un planteamiento más completo, flexible y general de la enseñanza de la lectura. Pero el empleo de estas listas de tareas como único criterio para asegurar el cumplimiento de objetivos es irracional.

Emplear estas listas de técnicas como criterio único conduce al segundo problema: la excesiva importancia concedida a la enseñanza de técnicas y la limitada variedad de lecturas que los niños realizan en la escuela. Cuando el éxito en la lectura se define en función de la puntuación obtenida en las pruebas estandarizadas, los profesores se preocupan más por enseñar las técnicas — «tareas definidas por las pruebas», como las denomina Popham (1983) - que por inculcar en los niños la satisfacción que la auténtica lectura proporciona.

\section{La lectura estética}

La lectura es mucho más que una lista de técnicas o una serie de preguntas de tests. Rosenblatt (1978), por ejemplo, escribe sobre la importancia de la lectura estética, es decir, la lectura escogida por el mero placer de la lectura, la lectura voluntaria de una novela o un poema. Como dice Frank Smith (1985, pág. 201):

"Con la lectura estética... nos resistimos a llegar al final. No leemos para obtener información, sino para explorar un mundo de sensaciones o ideas... Nos molesta que alguien intente privarnos de este tipo de lectura contándonos el final de la historia. A medida que nos acercamos a las páginas finales, reducimos la velocidad para prolongar la experiencia».

La lectura estética es lo que convierte a las personas en lectores motivados para toda la vida. Como no se concede importancia al gusto por la lectura dentro de los métodos actuales de cumplimiento cuantitativo de objetivos, nos preocupa que nuestros niños no lleguen a experimentar el placer estético de la lectura salvo en contadas excepciones. Paris, Lipson y Wixson (1983) resaltan que la lectura fluida «combina tanto técnica como voluntad". Nos preocupa que la concentración en la técnica vaya en detrimento de la voluntad.

En muchas aulas, los alumnos dedican de un sesenta a un setenta por cien del tiempo de lectura a realizar actividades que favorecen la adquisi- 
ción de dichas técnicas (Anderson, 1984; Fisher et al., 1978). Dicho de otro modo, los niños de los primeros cursos dedican aproximadamente una hora al día a rellenar fichas relacionadas con dichas técnicas y menos de $8 \mathrm{mi}$ nutos a la lectura silenciosa (Anderson et al, 1985). También se ha calculado que el $40 \%$ de los estadounidenses adultos capaces de leer libros eligen no hacerlo (Toch, 1984). Si coinciden con nosotros en que lo importante es formar lectores motivados, entonces la situación es deplorable. No obstante, nos cuesta criticar a los profesores por concentrar sus esfuerzos educativos en aquellos aspectos de la lectura sobre los que se les pedirá cuentas.

Queremos recalcar que nos preocupa tanto esta concepción tradicional que impone el cumplimiento cuantitativo de objetivos como la actitud que suele ir asociada a esa concepción. Es importante tener en cuenta esta actitud, puesto que aunque algunos estados, como Michigan (Wixson y Peters, 1984), estén desarrollando nuevos métodos de evaluación basados en una visión de la lectura más completa y compleja, estos esfuerzos, dignos de admiración, deben ir acompañados de una nueva actitud hacia la enseñanza de lectura y sus objetivos: una actitud que amplíe el curriculum en lugar de limitarlo, y que potencie el papel del profesor en lugar de restringirlo. Considerar la puntuación alcanzada en los tests la única medición legitima de un buen programa de lectura condiciona el clima en que tiene lugar la enseñanza. Es una actitud que impide que profesores y niños exploren los aspectos más importantes y gratificantes de la lectura y debe modificarse si realmente queremos mejorar la calidad de la enseñanza de la lectura.

\section{EL FRACASO PASIVO Y LA CONCEPCION DE LA LECTURA COMO ACTIVIDAD COMPETITIVA}

La segunda actitud que queremos examinar es la concepción de la lectura como actividad competitiva. Para apreciar en toda su extensión lo perjudicial de este planteamiento es importante revisar brevemente la relación existente entre la afectividad y la comprensión lectora.

Uno de los avances más importantes de las últimas investigaciones es la revaloración del papel de la motivación y la afectividad en la lectura estratégica. El contenido esencial de estas investigaciones revela que los buenos lectores son decididos, activos y flexibles, mientra que los lectores deficientes son indecisos, pasivos e inflexibles (véase Brown, 1982; Johnston y Winograd, 1985; Pans, Lypson y Wixson, 1983).

Los buenos lectores "emplean" la lectura para obtener información específica, para disfrutar o tal vez para escapar del aburrimiento y de la depresión. Por el contrario, muchos lectores pobres tratan de evitar la lectura o se concentran excesivamente en objetivos impropios, como «decir todas las palabras deprisa sin balbucear» (Canney y Winograd, 1979).

Los buenos lectores son capaces de orquestar distintas estrategias que les permiten alcanzar las metas propuestas. Los malos lectores, por el contrario, carecen del repertorio necesario de estrategias o no distinguen con claridad el tipo de estrategia más conveniente en cada situación, o se concentran en orquestar estrategias defensivas encaminadas a proteger el ego. 
Johnston y Winograd (1985) han acuñado el término "fracaso pasivo» para referirse a esta constelación de déficits afectivos, cognitivos o motivacionales.

El fracaso pasivo no es en absoluto la única causa de los problemas de lectura y de hecho puede ser una consecuencia de otros tipos de dificultades lectoras. En ambos casos tenemos pruebas suficientes para suponer que el fracaso pasivo interfiere en el desarrollo posterior de una buena estrategia lectora (Johnston y Winograd, 1985; Paris y Winograd, próxima publicación).

Además, creemos que la lectura entendida como una actividad competitiva contribuye al desarrollo del fracaso pasivo.

\section{Efectos negativos de la lectura entendida como actividad competitiva}

Uno de los rasgos que caracteriza la sociedad norteamericana es su espíritu competitivo (véase Henry, 1963). Disfrutamos compitiendo en el deporte, alabamos la competencia económica y a menudo competimos para medir la calidad de nuestra vida privada. Como Ellen Goodman (Abril, 1986) ha señalado, «en nuestra sociedad son muy pocos los que no han echado mano de las reglas para medir y contrastar su vida con la de sus amigos, incluso con la de sus mejores amigos».

Dada nuestra exagerada tendencia a la competitividad, no sorprende que los adultos hayamos llegado a considerar la lectura una actividad competitiva. Constantemente evaluamos a los alumnos para comprobar sus avances en habilidad lectora. Constantemente comparamos a unos con otros para identificar a aquellos que necesitan nuestra ayuda. Nuestras acciones son comprensibles; comparamos a los niños para agruparlos, porque una vez agrupados, la tarea de enseñarles a leer se simplifica.

Y si a nosotros nos cuesta abandonar esta visión de la lectura, a los malos lectores les resulta prácticamente imposible. La lectura no entraña más competitividad que escuchar música o charlar con un amigo, pero considerarla una competición acarrea problemas.

Cuando un niño empieza a medir su éxito comparando sus resultados con los que obtienen otros niños, le preocupa más evitar el fracaso y la vergüenza que aprender a leer. Y quizá comience a ser vulnerable a toda la gama de problemas de motivación y atribución, afectivos y cognitivos, implicados en el fracaso pasivo (Johnston y Winograd, 1985).

La distinción entre situaciones centradas en el ego y situaciones centradas en la tarea hecha por Nicholls es importante para comprender los efectos negativos de concebir la lectura como actividad competitiva.

En situaciones que involucran al ego, al sujeto le preocupa su actuación en comparación con la de otras personas. Si surgen dificultades, éstas son fuente de tensión, en especial cuando los demás parecen sobrellevar la situación sin mayores esfuerzos.

Por el contrario, las situaciones centradas en la actividad no son competitivas. Se realiza la actividad por el placer que se obtiene a cambio. Para muchas personas la jardinería es un ejemplo de este tipo de situaciones. Si surge algún problema, se afronta como un reto, no como una acusación por falta de habilidad.

Pero, en lugar de ser una situación centrada en la actividad la lectura, se 
convierte en una situación centrada en el ego cuando el éxito se define en función de la posición que uno ocupa respecto a los demás y no en función del cumplimiento de metas personales relevantes. Sin embargo, cuando el niño lee voluntariamente, mide su éxito o fracaso en una escala más personal y las consecuencias de las dificultades que puedan presentarse debilitan mucho menos el ego.

Nuestra concepción de la lectura como actividad competitiva puede que sea útil a la hora de organizar la enseñanza, pero consigue que muchos malos lectores consideren a aquélla como un concurso que nunca van a ganar. Lo que les conduce a la confusión, la frustración y el fracaso pasivo; algo que no lograremos cambiar mientras no modifiquemos nuestra actitud.

\section{LA ENSEÑANZA DE LA LECTURA Y EL ANQUILOSAMIENTO DE LOS PROFESORES}

Nos referiremos, por último, a la actitud desde la que se mantiene que el papel más importante del profesor de lectura es llevar a la práctica las decisiones educativas tomadas por las autoridades competentes. Shulman (1983) denomina este problema "el control remoto de la enseñanza».

Durkin $(1978-79)$ y Shannon $(1983,1984,1986)$ creen que la actividad educativa actual ha reducido al profesorado al papel de administradores de material. En concreto, Shannon ha escrito prolijamente sobre la excesiva dependencia que el profesor demuestra hacia los materiales de lectura.

Pero es fácil comprender esta dependencia. Cuando la lectura se divide en una serie compleja de técnicas menores, cada una de las cuales se evalúa de acuerdo con un calendario, se anula la capacidad de decisión del profesor sobre qué enseñar y cuándo hacerlo. Como explica Shannon (1983), "éste controla únicamente el nivel de precisión con que maneja el material».

Debido a este anquiloșamiento, el profesorado (Apple, 1982) se siente menos eficiente $\mathrm{y}$ profesional. Un reciente sondeo realizado por Harris (Freed, 27 abril, 1986), por ejemplo, revela que el aumento de la profesionalidad es la principal preocupación del profesorado.

Pero existe otro aspecto más sutil del anquilosamiento del profesorado que nos gustaría examinar: en qué medida afecta éste a las actitudes de los niños hacia la lectura.

Hemos oido comentar a varios profesores experimentados que la enseñanza de la lectura ha dejado de ser divertida. También hemos oído a nuevos y futuros profesores lamentarse por la complejidad de la enseñanza de la lectura y su miedo a cometer errores.

En nuestra opinión, el profesor aburrido o nervioso transmitirá esta sensación a los niños durante la clase. Csikszentmihalyi y McCormack (1986) expresan más elocuentemente esto mismo en un reciente artículo sobre la influencia del profesorado:

«Los muchachos no necesitan sólo información; necesitan información significativa. No necesitan conocimientos, sino conocimientos comprensibles que inspiren confianza... ¿Y cómo van a creer que la información que reciben merece la pena cuando el profesor parece aburrido, distante o indiferente? En la medida en que los profesores no se entreguen con interés a la enseñanza, sus esfuerzos serán prácticamente vanos». 
Sabemos muy poco sobre el efecto que tiene la actitud del profesor ante la enseñanza sobre la capacidad de aprendizaje del alumno. No obstante, se han realizado algunas investigaciones que pueden guiarnos en nuestras especulaciones.

Tomemos, por ejemplo, el trabajo de Tannen (1985) sobre la relevancia del discurso centrado en la participación. Según Tannen, hay tipos de discursos que se centran más en la participación, es decir, en la relación entre los participantes: por ejemplo, charlas informales o las notas que los alumnos se pasan en clase. Por el contrario, cuando el discurso se centra más en la información, lo importante es el contenido. Una conferencia o un texto de carácter técnico son ejemplos de discurso centrado en la información.

Creemos que para muchos niños, especialmente los más pequeños, la lectura es atrayente al principio, porque está centrada en la participación y en la información. Sólo hay que recordar lo insistentes que los niños pueden ser con los cuentos a la hora de acostarse para apreciar que la lectura a menudo favorece situaciones propicias para compartir momentos con otras personas.

Considérese, por ejemplo, este fragmento perteneciente al libro I know why the caged bird sings (Angelou, 1969). La protagonista, una niña llamada Marguerite Johnson, acaba de volver de visitar por primera vez a la Sra. Flowers, una de las damas más respetadas de la ciudad. Durante la visita, la Sra. Flowers lee a Marguerite un pasaje de Historia de dos ciudades. La reacción de Marguerite es la siguiente:

La lógica infantil no exige ser probada (todas las conclusiones son absolutas): «No pregunté por qué la Sra. Flowers me eligió a mí, ni se me ocurrió pensar que tal vez mami le había pedido que tuviera una charla conmigo. Lo único que me importó fue que había preparado pastas para mí y que me había leído a mí un fragmento de su libro favorito. Eso bastaba para probar que yo le gustaba.» (Angelou, 1969, pág. 85).

Nuestra preocupación es que el anquilosamiento lleve a los profesores a mecanizar la enseñanza de la lectura, como si el único fin fuera el de transmitir información, y que lleguen a olvidar que la lectura es un medio para que los niños disfruten de relaciones humanas y literarias importantes.

Estamos seguros de que un aspecto esencial del aprendizaje de la lectura es la compenetración que surge en el aula cuando los profesores dedican su tiempo a compartir el placer de la lectura con los alumnos. Pero no dedicarán ese tiempo si asumen que su tarea fundamental es seguir estrictamente las «lecciones, preguntas y actividades programadas» (Woodward, 1986).

\section{Referencias}

ANDERSON, LINDA (1984). The Environment of Instruction: The Function of Seatwork in a Commercially Developed Curriculum. En Comprehension Instruction: Perspectives and Solutions, editado por Gerald Duffy, Laura Roehler y Jana Mason, 93-115. Longman, Nueva York.

ANderson, Richard; Elfrieda Hiebert; Judith SCotT y IAN Wilkinson (1985). Becoming a Nation of Readers: The Report of the Commission of Reading, The National Institute of Education. Washington. 
ANGelou, MaYA (1969). I know Why the Caged Bird Sings, Bantam, Canadá.

Apple, MiChael (1982). Education and Power, Routledge and Kegan Paul, Boston.

Brown, ANn L.; Bransford, John D.; Ferrara, Roberta A. y Champione, Joseph (1982). Learning, Remembering, and Understanding, Informe Técnico número 244. University of Illinois at Champaign-Urbana, Center for the Study of Reading.

Canney, GeOrge y WinOgRand, PeTER (1979). Schemata for Reading and Reading Comprebesion Performance. Informe Técnico número 129. University of Illinois at ChampaignUrbana. Center for the Study of Reading.

Csikszentmihalyi, Mihaly y McCormack, Janet (1986). The influence of Teachers. Phi Delta Kappan, 67, 415-19.

DURKIN, DOLORES (1978-79). "What Classroom Observations Reveal about Reading Comprehension Instruction». Reading Research Quartely, 14 (4), 481-533.

Fisher, Charles; Berliner, David; Filby, Nikola; Marliave, Richard; Cohen, leoNARD; DISHAw, MARILYN; y MOORE, JEFFREY (1978). Theaching and Learning in Elementary School: A Summary of the Beginning Teacher Evaluation Study, Far West Laboratory for Research and Development, San Francisco.

FisKe, EDWARD, B. (1986), Effort to Upgrade Schools Shifts Focus to Motivation Work Climate, Lexington Herald-Leader, 27 abril.

FREED, JOHN C. (1986), Professionalism is Major Issue with Teachers, Lexington Herald-Leader, 27 abril.

Goodman, Ellen (1986), Setting into College Can Be Cruel Rife, Lexington Herald-Leader, 12 abril.

Harper, Robert J. y Kilarr, Gary (1978). Reading and the Law, International Reading Association, Newark.

HenRY, JULes (1963). Culture against Man, Random House, Nueva York.

JohNSTON, Peter y WinOGRAD, PETER (1985). Passive Failure in Reading. Journal of Reading Bebavior, 17 (4), 279-301.

NiCHOLLS, JOHN (1983). Conceptions of Ability and Achievement: A Theory and Its Implications for Education. En Learning and Motivation in the Clasroom, editado por Scott Paris, Gary Olson y Harold Stevenson, 211-37. Lawrence Erlbaum Associates, Nueva York.

Paris, Scott; MARge Lipson y Karen Wixson (1983), Becoming a Strategic Reader, Contemporary Educational Psychology, 8, 292-316, julio.

PARIS, SCOTT y WINOGRAD, PETER (En prep.). Meatacognition in Academic Learning and Instruction. En Dimensions of Thinking: A Review of Research, editado por Beau F. Jones, Association for Supervision and Curriculum Development.

POPHAM, W. JAMES (1983). Task-teaching Versus Test-teaching, Educational Measurement: Issues and Practice, 2 (4), 10-11.

RosenblatT, LOUISE (1978). The Reader, the Text, The Poem: The Transactional Theory of Literary Work, Southern Illinois University Press, Illinois.

ROSENSHINE, BARAK (1980). Skill Hierarchies in Reading Materials in American Elementary Schools. En Theoretical Issues in Reading Comprebension, publicado por Rand J., Spiro, Bertram C. Bruce y William F. Brewer, 535-54, Lawrence Erlbaum Associates.

Shannon, Patrick (1986). The use for Commercial Reading Materials in American Elementary School, Reading Research Quartely, 21 (2), 20-35.

Shannon, Patrick (1986). Mastery Learning in Reading and the Control of Teachers and Students, Language Arts, 61 (5), 484-93.

SHANNON, PATRICK (1986). Teacher's and Administrator's Thoughts on Changes in Reading Instruction within a Merit Pay Program Based on Test Scores. Reading Research Quartely, 21 (2), 20-35.

SHUlmAN, LeE (1983). Autonomy and Obligation: the Remote Control of Teaching. En Handbook of Teaching and Policy, publicado por Lee Schulman y Gary Skykes, 484-504. Longman, Nueva York.

SMITH, FRANK (1985), A Metaphor for Literacy: Creating Worlds or Shunting Information. En Literacy, Language, and Learning: The Nature and Consequences of Reading and Writing, editado por David Olson, Nancy Torrance y Angela Hildyard, 195-213. Nueva York, NY: Cambridge University Press.

TANNEN, DEBORAH (1985). Relative Focus on Involvement in Oral and Written Discourse. En Literacy, Language, and Learning: The Nature and Consequences of Reading and Writing, editado por David Olson, Nancy Torrance y Angela Hildyard, 124-47. Nueva York, NY: Cambridge University Press.

ToсH, THOMAS (1984), America's Quest for Universal Literacy. Education Week, vol. 4, n. ${ }^{\circ} 1$ (septiembre, 4), L3-L5.

Winograd, Peter, y Marilyn Greenlee (1986), Students Need a Balanced Reading Program". Educational Leadership, vol. 43 (febrero), 16-21.

WIXSON, KaREN, y CHARLES PETERS, Comprehension Assessment: Implementing an Interactive View of Reading. Educational Psychologist, en prensa.

Wixson, Karen, y ChARLES, Peters (1984), Reading Redefined: A Michigan Reading Association Position Paper. The Michigan Reading Joumal, vol. 17, 4-7. 
WoODWard, ARTHUR (1986), Over-programmed Materials: Taking the Teacher Out of Teaching. American Educator, vol. 10, n. ${ }^{\circ} 1,26-31$.

\section{Datos sobre el autor}

Winograd imparte cursos sobre lectura en la Universidad de Kentucky (134 Eastover, Lexington, Kentucky, 40502 U.S.A.) y su campo de investigación se centra en los factores implicados en las dificultades de comprensión de los malos lectores. Smith es estudiante de doctorado y enseña métodos de lectura en la misma universidad.

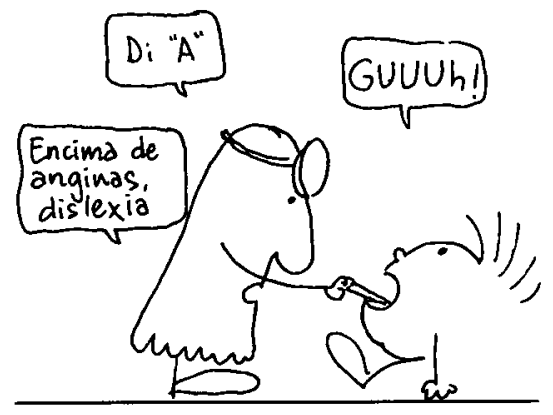

